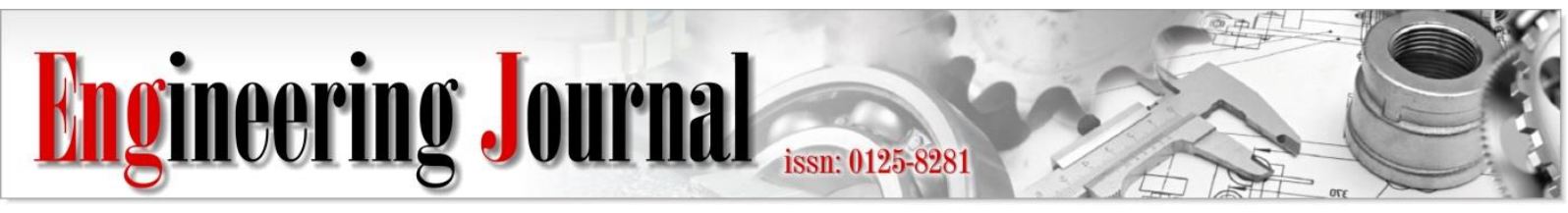

Article

\title{
A Study on the Single Caprylic Acid Fractionation and Centrifugal Separation of Equine Rabies Immunoglobulin
}

\author{
Issada Manohorratad ${ }^{a}$ and Angsumalin Senjuntichai ${ }^{\mathrm{b}, *}$ \\ Department of Industrial Engineering, Faculty of Engineering, Chulalongkorn University, Bangkok 10330, \\ Thailand \\ E-mail: a61704180@student.chula.ac.th, b,*angsumalin.s@chula.ac.th (Corresponding author)
}

\begin{abstract}
This study proposes alternative caprylic acid precipitation and centrifugal separation for the equine rabies Immunoglobulin manufacturing process. The objective is to determine the optimal setting associated with the centrifugal machine and the optimal amount of caprylic acid for the maximum process yield (\%). The experiments were designed based on the central composite design and performed to analyze the relationship of three factors which are the caprylic acid $(1 \%-5 \% \mathrm{~V} / \mathrm{V})$, the rotation speed $(7,500-12,500 \mathrm{rpm})$, and centrifugal time $(20-40 \mathrm{~min})$ on the yield of the process. For the first time, the prediction model as a second-degree polynomial regression is presented and developed by a response surface method (RSM) with $\mathrm{R}^{2}$ approximately $51 \%$. RSM model also reveals that the process yield is affected by the concentration of caprylic acid and the amount of time to centrifuge the precipitated plasma but not by the rotation speed of the centrifugal machine. With the predicted process yield of about $12.97 \%$, the optimal setting by RSM suggests the concentration of caprylic acid at $2.82 \%$ and the centrifugal time at 28 minutes.
\end{abstract}

Keywords: Equine rabies immunoglobulin, caprylic acid precipitation, centrifugal separation, central composite design, response surface method.

ENGINEERING JOURNAL Volume 25 Issue 11

Received 14 April 2021

Accepted 21 November 2021

Published 30 November 2021

Online at https:/ / engj.org/

DOI:10.4186/ej.2021.25.11.33 


\section{Introduction}

Aside from human rabies immunoglobulin (HRIG), there is also equine rabies immunoglobulin (ERIG) containing $\mathrm{F}\left(\mathrm{ab}^{\prime}\right)_{2}$ that provided acquired immunity and instantly neutralized rabies virus. Despite the fact that ERIG has more unfavorable side effects, it is less expensive than HRIG due to the larger volume of equine blood collected. As shown in Fig. 1(a), the Queen Saovabha Memorial Institute (QSMI) is Thailand's only ERIG manufacturing factory that uses sequential enzymatic digestion and fractionation with Ammonium Sulfate (AS) and Caprylic Acid (CA) in the precipitation step. Based on recent studies on the EGIR improvement, Santos et al. [1] and Perosa et al. [2] introduced a single CA into a non-igG precipitation and concluded that precipitation with CA alone may result in an increase in $\mathrm{F}\left(\mathrm{ab} \mathrm{b}^{\prime}\right)_{2}$, potency, and purity that outperforms a combination of AS and CA. Furthermore, caprylic acid precipitation has a stronger neutralizing effect. Rojas et al. [3] also proposed CA precipitation as a one step process optimization. Whereas Eursakun et al. [4] and Simsiwong et al. [5] investigated the fractionation of snake antivenom, using CA alone in the precipitation gives better overall results than the combination of AS and CA. Nonetheless, Eursakun et al. [4] and Simsiwong et al. [5] suggested different optimal CA percentages of 3.5 and 2, respectively, for snake anti-venom. Although the CA single precipitate is suggested, the optimal concentration varies from one literature to another.

Apart from $\mathrm{F}\left(\mathrm{ab}^{\prime}\right)_{2}$ and potency as product efficiency indicators, process yield is an intriguing process efficiency indicator that considers both the potency and volume of purified plasma. As a result, not only fractionation and precipitation are important in the ERIG process, but separation is also important for process improvement which has not been studied before until the recent study of the ERIG on the CA single precipitate followed by conventional filtration technique [6] in 2021. The filtration process [7] usually uses a filter press machine due to low cost, uncomplicated operation, and easy maintenance. The benefit of using a filter press is a large volume batch production that requires a large area of filtration and chamber space to store fractionated protein slurry. The filter presses use a filtration mechanism by trapping larger particles than the average pore size within the depth porous path of filter media packed in filter presses machine illustrated in Fig. 2. However, Eursakun et al. [4] reported that precipitation by $\mathrm{CA}$ alone increases the amount but decreases the size of precipitate, which causes a problem in the following separation step due to the mixture's high turbidity. The in-house study also discovered that the amount of precipitates from the single CA precipitation increased to $20 \% \mathrm{v} / \mathrm{v}$ with an average size of 50 micrometers. Milledge and Heaven [8] showed that the filtration method is appropriate for separating the amount and size of solid under $5 \% \mathrm{v} / \mathrm{v}$ and 1 micrometer, while centrifugal separation is preferable for the amount of solid between $2-25 \% \mathrm{v} / \mathrm{v}$ and the size of solid from 0.1 -
500 micrometers. Therefore, the centrifugal separation is proposed for the first time as an alternative method of ERIG separation to the filtration method. In this technique, the solid and liquid are separated by radial centrifugal forces (RCF) generated while the mixture is centrifuged as shown in Fig. 3.

Therefore, to increase the ERIG potency and volume together, this study proposes the single CA precipitation for ERIG Manufacturing along with the alternative centrifugation method for the first time to separate a high content and a large amount of precipitate that resulted from the single CA precipitation shown in Fig. 1(b). As a result, firstly, the experiments will be proposed and performed for different levels of the CA concentration in the precipitation and of the centrifugation parameters in the separation. Secondly, the prediction model will be constructed from the experimental results to examine the effect of the CA concentration in alignment with the centrifugation parameters on the process yield/recovery. Finally, the optimal process settings will be determined concerning the highest process yield.

The response surface methodology (RSM) [9] is a well-known method for process optimization applications. RSM is useful for exploring the relationship between the interesting response and the associated factors based on experimental data wildly applied in many engineering applications [10-14]. Most RSM applications use the second-order model concerning the main, quadratic, and interaction effect between response and factors. It needs fewer experiments to study the effects of all the factors and the optimum combination of all the variables can be revealed. To find the optimum conditions for all variables, RSM uses the steepest ascent (or descent) [9] to find the next local optimum until it approaches the optimum. Recently, there have been studies on the integration of heuristic methods such as Genetic Algorithm, Simulated Annealing [15, 16] or the Heuristic Response Surface method [17] to improve the optimum efficiency. However, the combined heuristic methods need more experimental runs than traditional RSM and are also preferable for more than 3 factors and higher-order (cubic) response models. Compared to those heuristic methods, RSM is easier to implement, understand, and interpret, theoretically and practically. In addition, RSM, which needs fewer numbers of experimental runs, is preferred for the high cost experiments of ERIG. With the successful applications of RSM in a number of research suggestions, it appears to be the best choice for this study.

The objective of this study is to determine the optimal concentration of CA in the precipitation step and the optimal setting for the centrifugal separation parameters which give the highest yield based on the experimental design (DOE) using RSM. DOE is a powerful method in discovering the optimal process conditions for different fields of application, for instance, biochemical process [6], industrial engineering $[12,13]$, chemical process $[14,18]$, biodiesel [10,11], autonomous vehicle [19], and solid fuel [20]. The designs such as general factorial $[12,19,20]$, central composite (CCD) [18], and Box-Behnken [6, 10, 
$11,13,14,18]$ designs vary from application to application due to the research objective and limitation.

The paper organizes as follows: the second section details the ERIG manufacturing process of QSMI, while the explanation of the applied methodology, material, and experimental design is in the third section. The experimental results are analyzed and discussed in section 4. The final section shows the conclusions and suggestions for future works.

\section{ERIG Manufacturing Process}

Figure 1(a) presents the summary of the current ERIG manufacturing process [21]. Equine immunization uses veterinary rabies vaccine (Rabisin $\left.{ }^{\circledR}\right)$ and Freund's Complete Adjuvant in the CEP production. Production bleeding is done by plasmapheresis. In the first process called CEP pooling, individual CEP is pooled and mixed. The pooled CEP dilutes with a double volume of water for injection. The next step is pepsin digestion to breakdown the disulfide bond of immunoglobulin and cleaves $\mathrm{F}\left(\mathrm{ab}^{\prime}\right) 2$ and $\mathrm{FC}$ regions. The CEP adjustment is applied by adding pepsin enzyme (the quantity depends on the total amount of protein in pooled CEP) and carried out for 30 minutes. The third step is thermo-coagulation that can precipitate unwanted plasma protein by adding AS to mix with the digested plasma is then added and mixed for 60 minutes. The fourth step is precipitation with the $\mathrm{CA}$ to precipitate retained unwanted protein. In this step, CA is added and mixed with the thermo-coagulated plasma for 60 minutes. In the fifth step of filtration and diafiltration, the slurry precipitated plasma is filtered by filter press to discard the unwanted protein which can be the cause of the adverse reaction. After that, the filtrate which contains $\mathrm{F}\left(\mathrm{ab}^{\prime}\right) 2$ is adjusted and diafiltrated to eliminate AS and CA residues and concentrates $\mathrm{F}(\mathrm{ab}) 2$. The last step is the formulation and filling process in which the concentrate $F\left(a b^{\prime}\right) 2$ is formulated to require a potency of $200 \mathrm{IU} / \mathrm{mL}$ and osmolarity of $240-400$ $\mathrm{mOsm} / \mathrm{kg}$. Finally, $\mathrm{F}\left(\mathrm{ab}^{\prime}\right) 2$ is sterilely filtrated and filled in the bottle as the finished products. The plasma in each step must be adjusted to the desired temperature and $\mathrm{pH}$ with specified hydrochloric acid or sodium hydroxide.

The potency is the measure of the virus-neutralizing antibodies titer in the unit of $\mathrm{IU} / \mathrm{ml}$. However, the efficiency of the ERIG production depends on the potency and volume produced from the process. Therefore, the efficiency is measured as process yield (percentage) and calculated as described in Eq. (1).

$$
\text { Yield }=\frac{\text { Potency of Conc. } F\left(a b^{\prime}\right) 2 \times \text { Vol. of Conc. } F\left(a b^{\prime}\right) 2}{\text { Potency of Pooled CEP } x \text { Vol. of Pooled CEP }} \times 100
$$

The previous study of snake anti-venom toxin preparation applies the same production step as the AS in the thermo-coagulation that could increase the loss of $\mathrm{F}\left(\mathrm{ab} \mathrm{b}^{\prime}\right) 2$ due to the increase of the precipitation of $\mathrm{F}\left(\mathrm{ab}^{\prime}\right) 2$ and affect the secondary and tertiary structure of $\mathrm{F}(\mathrm{ab}) 2$ as well [4]. The AS can decrease the precipitation of plasma protein by CA due to the salting effect [4]. In comparison with AS in the precipitation step, CA precipitates most of the plasma protein except $\mathrm{F}\left(\mathrm{ab}^{\prime}\right) 2$ [4]. Besides, CA can inactivate the virus that may present in the CEP [22]. After the precipitation step, an increase in the $\mathrm{CA}$ concentration increases the mixture turbidity that affects the filtration step [4]. But, the presence of AS can gradually decrease the turbidity generated in precipitation with CA. The study of Eursakun et al. [4] suggested that using CA alone at 3.5\% seems to yield the optimal results in consideration of turbidity, activity recovery, and purification. The conclusion from Simsiwong et al. [5] revealed that using CA alone at $2 \%$ has the benefit over the combination of AS and CA. While, Intarapalit and Senjuntichai [6] recommended $1 \%$ for the CA concentration for ERIG. However, Using CA alone has an essential drawback. It can cause problems in the filtration steps due to the high turbidity of the mixture. Therefore, the separation process between the precipitated plasma and unwanted protein should be concerned.

In centrifugal separation, the solid and liquid are separated by radial centrifugal forces generated while the mixture is centrifugal. The RCF drives the solid to accumulate together away from the axis of rotation and settling the firm cakes. The setting rate of the firm-cakes depends on the radial centrifugal force and the soliddensity. The higher density or larger solid-size is typically setting the firm cake faster than the less dense or smaller. The modified Stoke's law [23] explains that the cake settling depends directly on RCF. The RCF itself is expressed relative to the earth's gravitational forces and exponentially related to the rotation speed (RS, revolution per minute) shown in Eq. (2) [24].

The RCF is essential for cake settling. The $r_{m m}$ (the rotational radius, $\mathrm{mm}$ ), and $\mathrm{RS}$ as one of the centrifugal parameters are under the consideration in this study. The $r_{m m}$ as the rotational radius of the centrifugal machine depends on the machine model. Then it is considered as a constant for this study.

$$
R C F=1.118 \times 10^{-6} r_{m m} R S^{2}
$$

Meanwhile, the mixture consists of a wide-size distribution of the solid. The centrifugal time (CT) needs to be sufficient for the low-density solid with a low moving speed to the cake settling area. Therefore, CT becomes one of the crucial parameters.

Figure 1(b) shows the proposed process with single precipitation of CA and the centrifugal separation instead of the filtration compared to the QSMI current process. The objective is to determine the optimal concentration of CA and the optimal setting of the centrifugal separation parameters, CT and RS, by designing the experiments. 
a)

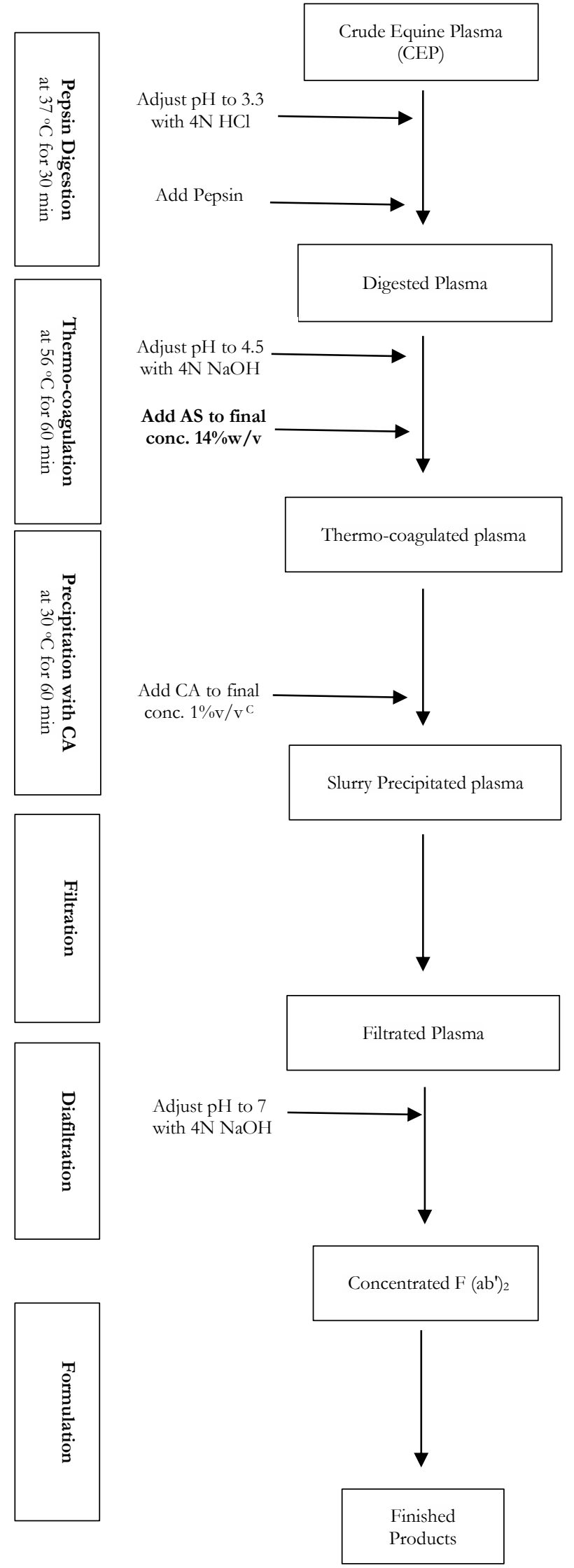

b)
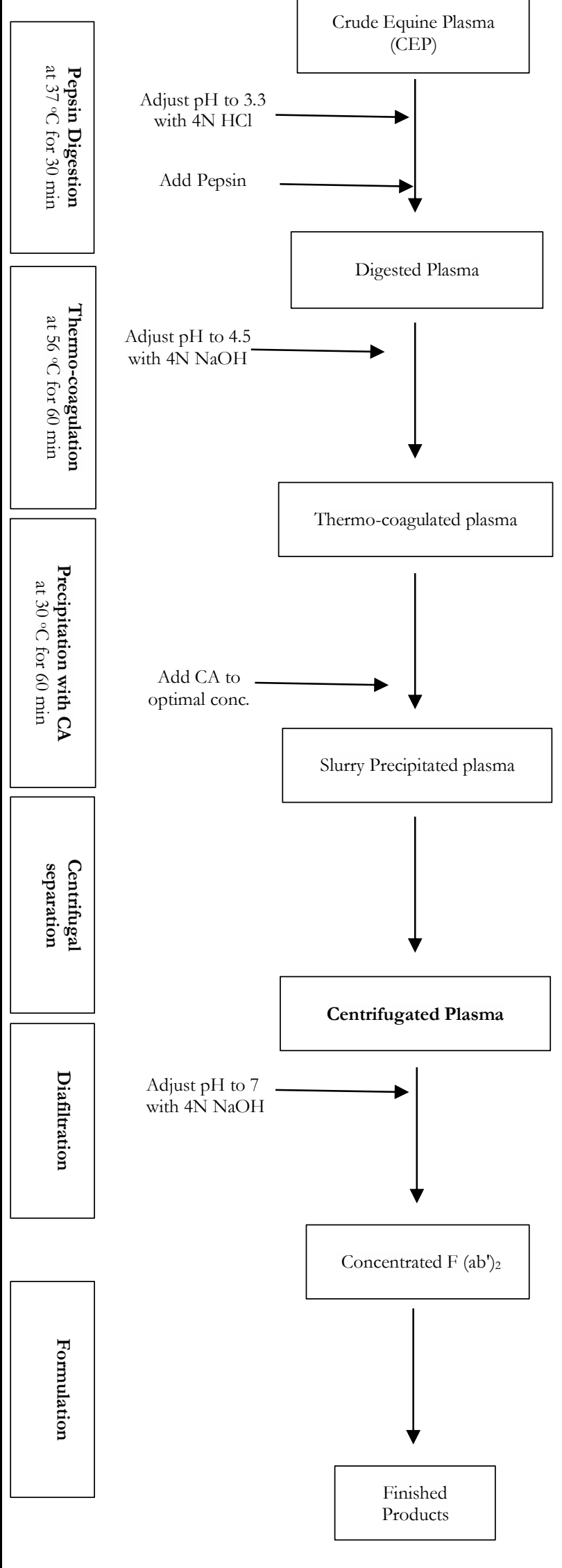

Fig. 1. The ERIG manufacturing process: (a) Current Process by QSMI (b) Proposed process using CA alone and centrifugal separation instead of filtration. 


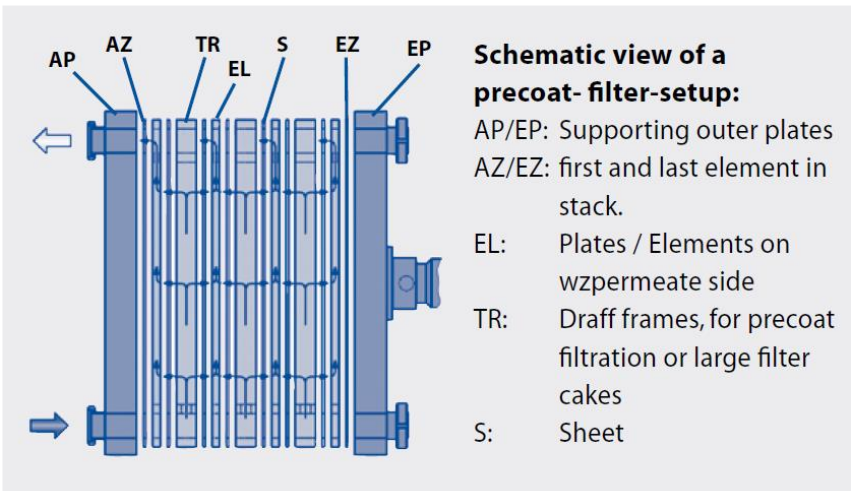

Fig. 2. Filter press component.

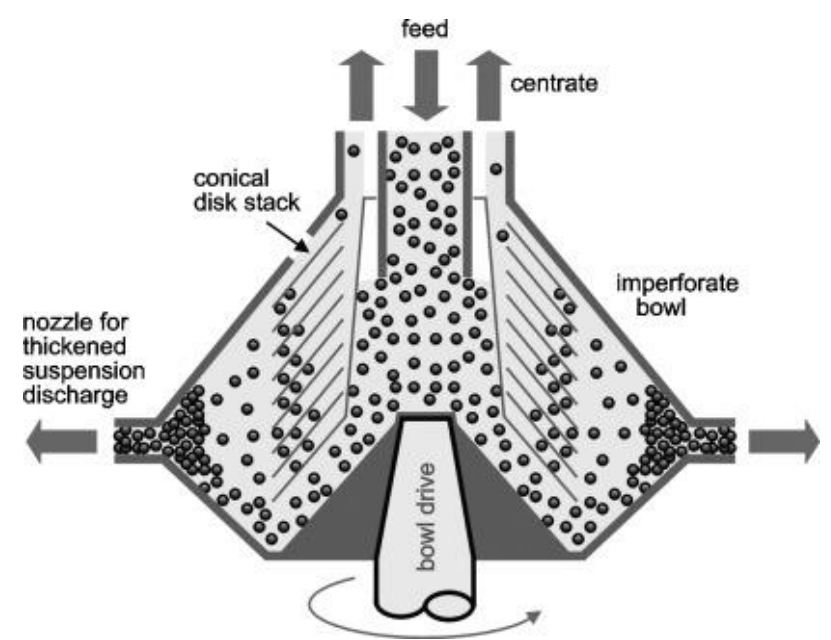

Fig. 3. Solid-Liquid Centrifugal Separation.

\section{Material and Methods}

\subsection{Chemicals and Biochemicals}

Chemical, biochemical, and digested plasma were pharmaceutical grades and sponsored by the QSMI. The digested plasma was prepared with the same process as the ERIG Manufactured by the QSMI.

\subsection{Factors, Levels and Response Variables}

In this study, there are three crucial factors, CA, CT, and RS. CA is the concentration (\%) of caprylic acid used in the precipitation with the CA step. CT and RS are associated with the centrifugal process. Referring to the previous study [4-6], the CA setting was between 1$5 \% \mathrm{~V} / \mathrm{V}$. According to the rotational radius of $8.5 \mathrm{~cm}$. of the centrifugal machine model, Hermle Z383K, using in this study, the RS was set between 7,500-12,500 rpm resulting in RCF around 11,000 $\mathrm{g}$ as reported by Santos et al. [1]. With the preliminary studies of the QSMI, the CT setting was between 20-40 minutes. The process performance measurement is the process yield (\%) as the response of the study.

\subsection{Experimental Design}

With three factors and three levels, there are three experimental designs called $1.3^{3}$ Factorial Design, 2. BoxBehnken design (BBD) and 3. Central Composite Design $(\mathrm{CCD})$ that are useful for constructing the second-degree prediction model $[9,25]$. Among the three designs, factorial design requires the maximum number of experiments (27 experiments) and lacks rotatability. BBD requires the smallest number of experiments (15 experiments) and provides rotatability but it gives the worse quality of the mathematical model in comparison to CCD due to a lack of combined high-level experiments [18]. Whereas CCD provides the best mathematical model and requires a few more number experiments (2 more) compared to BBD. CCD consists of 3 types of experiments, i.e., $2^{\mathrm{k}}$ full factorial experiment (as shown by blue circle), $2 \mathrm{k}$ axial experiment with the axial distance of $\alpha$ from the center point (illustrated by green star) and $n$ replication of center point experiments (presented by red cross) as shown in Fig. 4, where $\mathrm{k}$ is a number of factors [26-28]. The CCD is made rotatable that indicates the consistent and stable variance of predicted response at points of interest [29] with the choice of $\alpha$ that depends on the number of factors involved. Therefore, CCD requires five different levels for each factor. With three factors for this study, CCD requires 14 experiments (with a 1.682 value of $\alpha$ ) plus at least three center point replication. By performing this design, the study of the quadratic and interaction effects of two factors on a response variable is under the consideration. Table 1 shows the setting levels of experimental factors. The lowest and the highest values of each factor are calculated with a 1.682 axial distance from the center point of 0 . Table 2 presents the experiment matrix based on CCD with two more replications at the axial point for a concentration of caprylic acid as the most crucial factor.

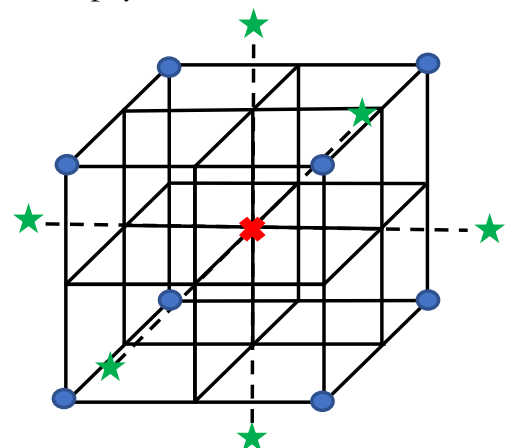

Fig. 4. Central Composite Design.

Table 1. The tested levels of experimental factors.

\begin{tabular}{lcccccc}
\hline \multirow{2}{*}{ Factor } & \multirow{6}{*}{ Unit } & \multicolumn{6}{c}{ Coded Levels and Corresponding Absolute Levels } \\
\cline { 3 - 7 } & & $\begin{array}{c}-\boldsymbol{\alpha} \\
\mathbf{( - 1 . 6 8 2 )}\end{array}$ & $\mathbf{- 1}$ & $\mathbf{0}$ & $\mathbf{1}$ & $\begin{array}{c}\boldsymbol{\alpha} \\
\mathbf{1 . 6 8 2})\end{array}$ \\
\hline $\mathrm{CA}$ & $\% \mathrm{v} / \mathrm{v}$ & 1 & 1.81 & 3 & 4.18 & 5 \\
\hline $\mathrm{RS}$ & $\mathrm{rpm}$ & 7,500 & 8,513 & 10,000 & 11,486 & 12,500 \\
\hline $\mathrm{CT}$ & $\mathrm{min}$ & 20 & 24 & 30 & 35 & 40 \\
\hline
\end{tabular}

\subsection{Thermo-coagulation and Precipitation with CA}

The digested plasma was thermo-coagulated by stirring for 60 minutes while the temperature was adjusted and controlled to $56^{\circ} \mathrm{C}$. The $\mathrm{pH}$ and temperature of Thermo-coagulated plasma adjust to 5.4 with $4 \mathrm{~N} \mathrm{NaOH}$ and $30^{\circ} \mathrm{C}$, respectively. Thermo-coagulated plasma is 
following divided into five parts. CA is added to each part for a final concentration of $1 \%, 1.81 \%, 3 \%, 4.18 \%$, and $5 \%(\mathrm{~V} / \mathrm{V})$. The increasing concentration of CA gradually increases the amount of the precipitated shown in Fig. 5. The precipitated plasma divides into 21 centrifugal tubes. Each tube contains $30 \mathrm{ml}$ of precipitated plasma.

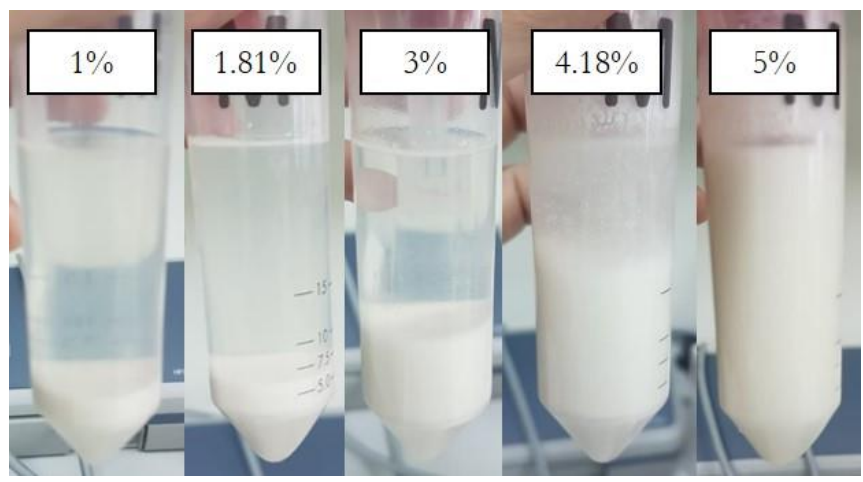

Fig. 5. The precipitated plasma with different CA concentration.

\subsection{Centrifugation}

The precipitated plasma was centrifuged to separate unwanted protein. The centrifugation was performed by Hermle Z383K illustrated by Fig. 6 with a constant $8.5 \mathrm{~cm}$ of the $r_{\mathrm{mm}}$ at rotation speed for an amount of time for each experiment shown in Table 2.

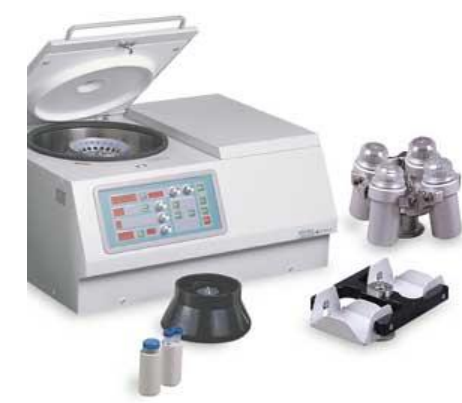

Fig. 6. Laboratory Centrifuge Hermle Z383K.

\subsection{Potency measurement and Yield calculation}

The QSMI laboratory measures the CEP potency and the centrifugated plasma by using the Rapid Fluorescence Focus Inhibition test. Each sample was diluted two-fold with medium supplemented with $2 \%$ fetal bovine serum and placed in a 96-well microplate. The rabies virus was added to each well and incubated in a 5\% CO2 incubator at $37^{\circ} \mathrm{C}$ for 90 minutes. Baby hamster kidney- 21 cells were added to each well and incubated for 24 hours. Finally, the baby hamster kidney -21 monolayer was fixed with $80 \%$ $\mathrm{v} / \mathrm{v}$ acetone and stained with a fluorescent antibody to detect the presence of a non-neutralized virus (fluorescent foci) under a fluorescence microscope. The Rapid Fluorescence Focus Inhibition test is applied to determine a titer of virus-neutralizing antibody (IU/ml). An IU denotes an international unit calculated from the titer by comparison with the WHO international standard of antirabies immune globulin.

Equation (3) presents the formula for the process yield as previously described in Eq. (1) by using the potency of the centrifugated plasma instead of the potency of the concentrated $\mathrm{F}(\mathrm{ab}) 2$. The potency of the CEP samples for this study measured at the beginning of the experiment was $98.97 \mathrm{IU} / \mathrm{ml}$ of the CEP potency. All experiments were performed with the same CEP when each tube contains $30 \mathrm{ml}$. of precipitated plasma. The potency and volume of pooled CEP used in Eq. (3) are $98.97 \mathrm{IU} / \mathrm{ml}$, and $30 \mathrm{ml}$., respectively, for all trials.

$$
\text { Yield }=\frac{\text { Potency of Centri. } x \text { Vol. of Centri. }}{\text { Potency of CEP } x \text { Vol. of CEP }} \times 100 \text { (3) }
$$

\section{Results and Discussion}

Table 2 presents the experimental matrix and results. The process yield as the response of the analysis is calculated following Eq. (3) and shown in Table 2. Based on the experimental results, CA, RS, CT, and yield are used for constructing the regression model and analysis.

A least square method is used to derive a mathematical correlation by fitting a response surface $[9,25]$ to measure the yield at specific points of the experimental design matrix. The response surface methodology is a powerful method for process optimization and improvement wildly applied by many engineering applications [10-14, 18-20] in exploring the relationship between the interested response and the associated factors. Equation (4) shows the seconddegree equation concerning the main $\left(x_{i}\right)$, quadratic $\left(x_{i}^{2}\right)$, and interaction $\left(x_{i} x_{j}\right)$ effect between response and factors [29]. Table 3 illustrates the result for regression analysis.

$$
\begin{aligned}
\text { Yield }= & -11.3+1.68 C A+0.00047 R S+1.30 C T \\
& -0.933 C A^{2}+0.00 R S^{2}-0.0216 C T^{2} 0.109(C A \times C T) \\
& +0.000053(C A \times R S)-0.000041(R S \times C T)
\end{aligned}
$$

This complete model predicts yield based on all three linear, all three quadratics, and all two-way interactions between the studied factors. The model F-value of 1.4 from the Analysis of Variance with a p-value of 0.296 indicates that the model equation is not beneficial in predicting the process yield. The difference between $\mathrm{R}^{2}$ of $53.33 \%$ and $\mathrm{R}^{2}$ adj of $15.15 \%$ also shows that the complete model overfitted and resulting in some non-useful predictors.

With a $5 \%$ significance level, all terms but $\mathrm{CA}^{2}$ with a $\mathrm{p}$ value higher than 0.05 are not useful in predicting the process yield. Therefore, to enhance the model, some of those non-useful terms will be removed from the analysis. There are two approaches for predictor selection, i.e., best subset approach and stepwise approach. The best subset approach aims to find the best mathematical model (the model with a maximum $\mathrm{R}^{2}$ adjusted or minimum mean squared error) by comparing all possible models using a specified set of predictors. Alternatively, the stepwise approach aims to find the subset without significant loss of information at specific statistical significance. 
Table 2. Experimental matrix and results.

\begin{tabular}{|c|c|c|c|c|c|c|c|c|}
\hline No. & CA & RS & CT & $\begin{array}{l}\text { Potency of } \\
\text { CEP } \\
\text { (IU } / \mathrm{ml} .)\end{array}$ & $\begin{array}{l}\text { Volume of } \\
\text { CEP } \\
\text { (ml.) }\end{array}$ & $\begin{array}{c}\text { Potency of } \\
\text { Centrifugated Plasma } \\
(\mathrm{IU} / \mathrm{ml} .)\end{array}$ & $\begin{array}{c}\text { Volume of } \\
\text { Centrifugated } \\
\text { Plasma (ml.) }\end{array}$ & $\begin{array}{c}\text { Yield } \\
(\%)\end{array}$ \\
\hline 1 & 3.00 & 10,000 & 40 & 98.97 & 30 & 15.35 & 16.5 & 8.53 \\
\hline 2 & 5.00 & 10,000 & 30 & 98.97 & 30 & 28.46 & 10.5 & 10.07 \\
\hline 3 & 3.00 & 7,500 & 30 & 98.97 & 30 & 14.8 & 25.0 & 12.46 \\
\hline 4 & 4.18 & 8,513 & 24 & 98.97 & 30 & 13.59 & 20.0 & 9.15 \\
\hline 5 & 1.81 & 8,513 & 35 & 98.97 & 30 & 13.59 & 24.5 & 11.21 \\
\hline 6 & 3.00 & 10,000 & 30 & 98.97 & 30 & 15.45 & 20.0 & 10.41 \\
\hline 7 & 1.00 & 10,000 & 30 & 98.97 & 30 & 13.53 & 13.0 & 5.92 \\
\hline 8 & 1.00 & 10,000 & 30 & 98.97 & 30 & 15.45 & 23.5 & 12.23 \\
\hline 9 & 4.18 & 11,486 & 24 & 98.97 & 30 & 14.13 & 22.0 & 10.47 \\
\hline 10 & 1.81 & 8,513 & 24 & 98.97 & 30 & 14.59 & 22.5 & 11.06 \\
\hline 11 & 5.00 & 10,000 & 30 & 98.97 & 30 & 15.39 & 17.5 & 9.07 \\
\hline 12 & 5.00 & 10,000 & 30 & 98.97 & 30 & 16.28 & 13.0 & 7.13 \\
\hline 13 & 1.00 & 10,000 & 30 & 98.97 & 30 & 14.07 & 19.5 & 9.24 \\
\hline 14 & 3.00 & 10,000 & 30 & 98.97 & 30 & 19.21 & 23.5 & 15.20 \\
\hline 15 & 4.18 & 8,513 & 35 & 98.97 & 30 & 14.23 & 24.0 & 11.50 \\
\hline 16 & 3.00 & 10,000 & 20 & 98.97 & 30 & 21.15 & 17.5 & 12.47 \\
\hline 17 & 3.00 & 10,000 & 30 & 98.97 & 30 & 14.80 & 25.0 & 12.46 \\
\hline 18 & 1.81 & 11,486 & 35 & 98.97 & 30 & 14.02 & 23.0 & 10.86 \\
\hline 19 & 4.18 & 11,486 & 35 & 98.97 & 30 & 16.00 & 22.5 & 12.13 \\
\hline 20 & 1.81 & 11,486 & 24 & 98.97 & 30 & 16.28 & 23.0 & 12.61 \\
\hline 21 & 3.00 & 12,500 & 30 & 98.97 & 30 & 17.16 & 22.5 & 13.00 \\
\hline
\end{tabular}

Table 3. Results for regression analysis.

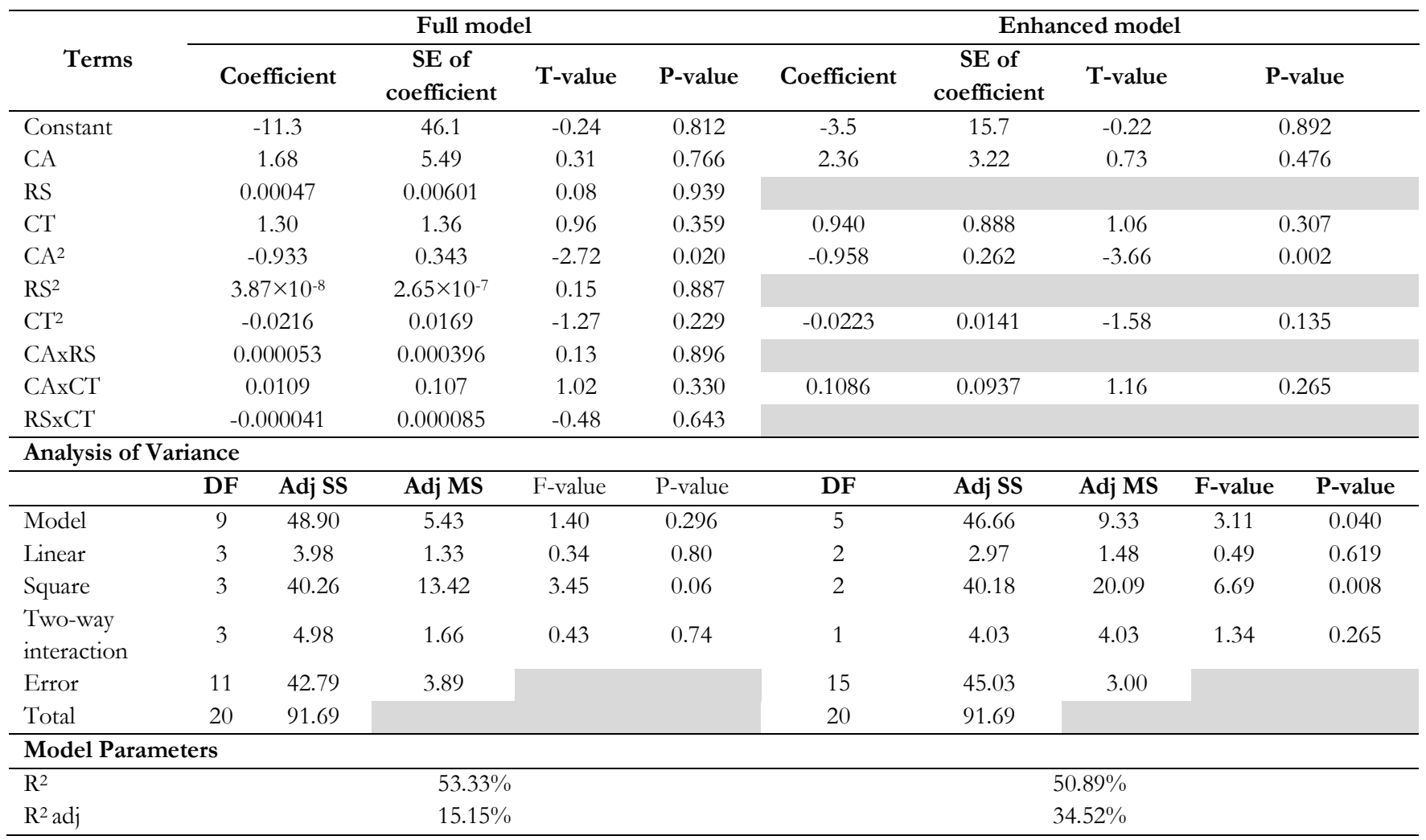


For the stepwise approach, the model is selected by adding or removing one individual predictor at a time based on their statistical significance. Since the best subset approach gives the prediction more accurate than the stepwise approach, the mathematical model was enhanced by this approach. All subset models consisting of the models with one term, two terms, so on will be compared according to $\mathrm{R}^{2}$ adj. The hierarchical model with the highest $\mathrm{R}^{2}$ adj is selected as shown in Eq. (5).

$$
\begin{aligned}
\text { Yield }= & -3.5+2.36 C A+0.940 C T-0.958 C A^{2} \\
& -0.0223 C T^{2}+0.1086 C A \times C T
\end{aligned}
$$

The enhanced hierarchical model consists of the linear term of CA and CT, the quadratic terms of CA and CT, and the interaction term of $\mathrm{CA} \times \mathrm{CT}$. In comparison to the full model described in Eq. (4), the F-value of the enhanced model from the Analysis of Variance increases to 3.11 with a p-value of 0.04 indicates that the enhanced model is useful in predicting the process yield at a $5 \%$ significance level

The $\mathrm{p}$-values of the t-test for the significance of individual terms show that only the second-order of CA with a p-value of 0.002 is statistically significant in predicting the process yield at the same $5 \%$ significance level. However, the $\mathrm{R}^{2}$ adj increases from $15.15 \%$ to $34.52 \%$. Therefore, the enhanced model is significantly useful and better than the full model. Although, the performance of the enhanced model represented by the $\mathrm{R}^{2}$ decreases from $53.33 \%$ to $50.89 \%$ but the difference between the $\mathrm{R}^{2}$ and $\mathrm{R}^{2}$ adj of the enhanced model is smaller than those of the full model.

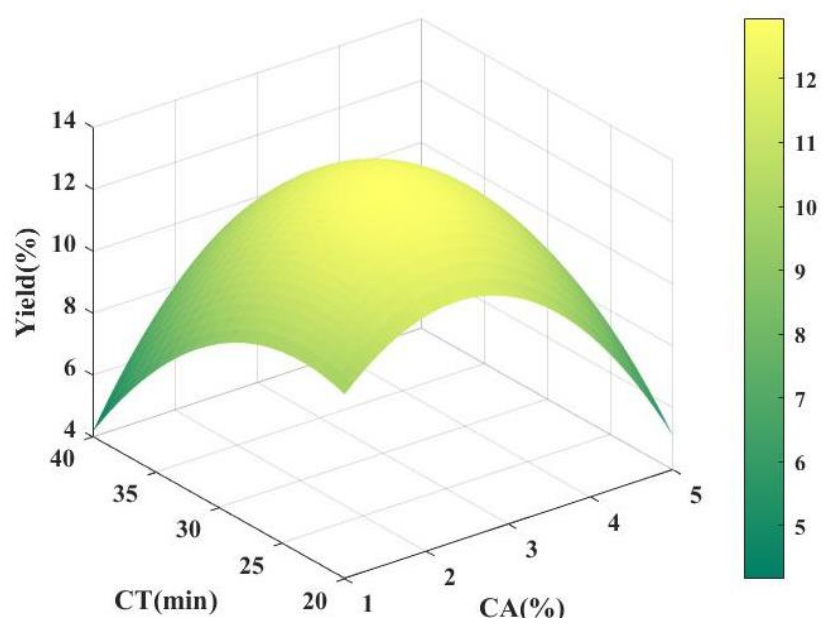

Fig. 7. Surface plot of Yield vs CA and CT.

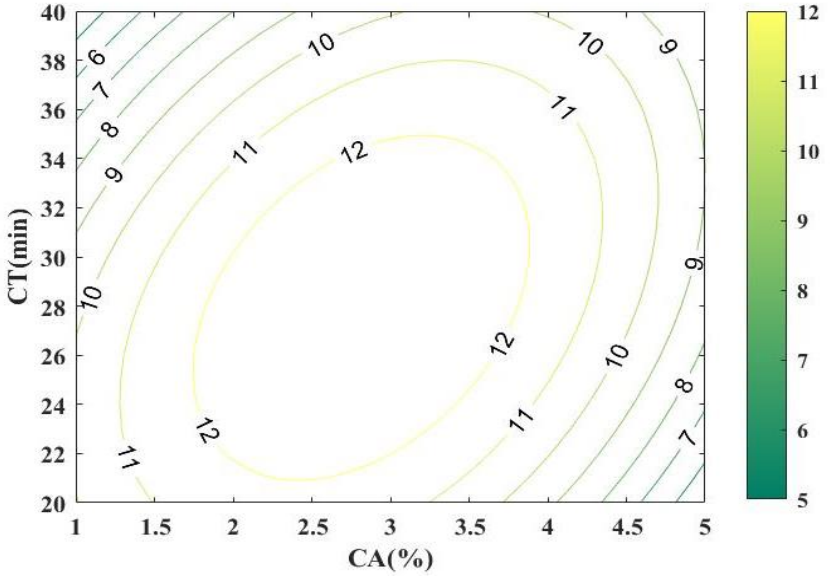

Fig. 8. Contour plot of Yield vs CA and CT.

Based on the enhanced model, the process yield is affected by the concentration of caprylic acid (\%) and the centrifugal time but not by the rotation speed. The amount of centrifugal time used in this experiment (20-40 min) may be long enough for settling the firm cake no matter which rotation speed (between 7,500-12,500 rpm) can be. With the negative quadratic terms of $\mathrm{CA}$ and $\mathrm{CT}$, the process yield is parabolically declining if $\mathrm{CA}$ or $\mathrm{CT}$ increases. On the other hand, shown by the positive linear and two-way interaction terms of CA and CT, the process yield is larger if $\mathrm{CA}$ or $\mathrm{CT}$ increase. The magnitude of +2.36 of the CA coefficients plus +0.1086 of the CAXCT coefficient together seems to be larger than the magnitude value of -0.958 of the $\mathrm{CA}^{2}$ coefficient, but the range of $\mathrm{CA}$ from $1-5 \%$ makes $\mathrm{CA}^{2}$ term is more contributed to yield than the CA term. Therefore, the concentration of caprylic acid (\%) alone has a positive effect on the yield of the process for a small value of $\mathrm{CA}$ as illustrated by Fig. 7, the surface plot of the process yields versus CA and CT. The process yield increases when the concentration of $\mathrm{CA}$ increases from 1 to almost $3 \% \mathrm{~V} / \mathrm{V}$ and declines afterward. Even though the value -0.0223 of the $\mathrm{CT}^{2}$ coefficient is smaller in magnitude than the value +0.940 and +0.1086 of the CT and CAXCT coefficient, respectively, but the CT range from 20-40 minutes makes the centrifugal time negatively effects the process yield when CT gets larger. The same as the CA concentration, Fig. 7 shows that increasing CT from 20 to almost 30 minutes increases the yield, the increasing CT from 30 to $40 \mathrm{~min}$ decreases the process yield. Figure 8 shows the contour plot with the light area in the center, in which the concentration of CA is from 2.0 to $3.5 \% \mathrm{~V} / \mathrm{V}$ and $\mathrm{CT}$ is from 22 to 32 minutes, gives the highest yield of more than $12.0 \%$. Note that residuals of the data used for regression have been checked to have an approximately normal distribution with zero mean, common variance, and be independent of one another. 


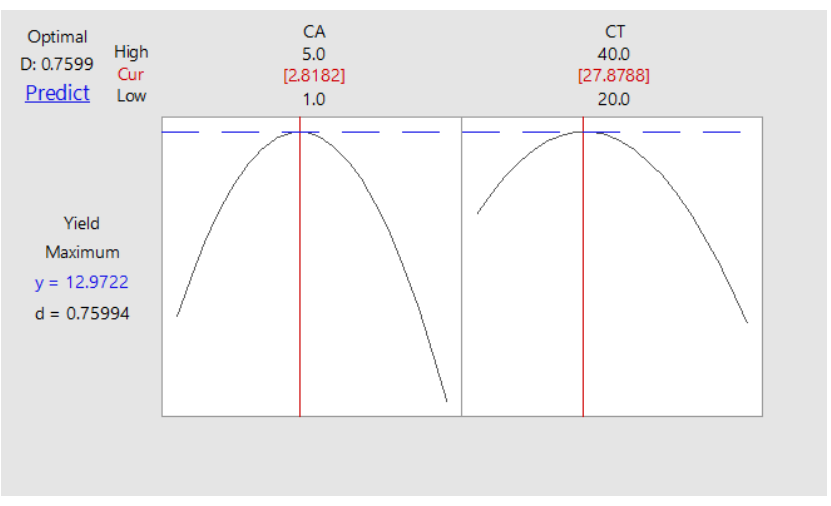

Fig. 9. Optimization plot for the maximum Yield.

Finally, the regression equation shown in Eq. (5) was employed to determine the optimal setting of CA and CT concerning the maximum process yield. Figure 9 demonstrates the optimization plot of the highest process yield of $12.97 \%$ when CA and CT are set at $2.82 \%$ and 27.88 (rounded up to 28) minutes, respectively. Comparing to $1 \%$ CA concentration with the filtration method suggested by Intarapalit and Senjuntichai [6], the $2.82 \%$ CA concentration with centrifugal technique precipitate other unwanted protein better [4, 5, 29] and more thoroughly [8]. The Centrifugal technique is more suitable with finer sludge. The size of protein sludge precipitate from higher CA concentration is also more varied [8] in which the centrifugal method can separate better than filter press with fixed pore size membrane.

\section{Conclusions}

Despite recent research in the product improvement on ERIG, this paper proposed a product and process improvement for the ERIG manufacturing process. According to the suggestion of using only caprylic acid instead of using the combination of ammonium sulfate in the thermo-coagulation process followed by caprylic acid precipitation, the objective of this paper is to determine the optimal setting associated with the centrifugal machine as well as the optimal amount of caprylic acid used for the maximum process yield. The experiments based on the central composite design were applied to analyze the relationship of three factors, the caprylic acid $(1 \%$ $5 \% \mathrm{~V} / \mathrm{V})$, the rotation speed $(7,500-12,500 \mathrm{rpm})$, and centrifugal time (20-40 min) on the process yield (\%). For the first time, a mathematical equation presenting the relationship between the process yield of ERIG with the CA concentration and centrifugal time is developed for predicting the ERIG process yield.

The best subset approach by the response surface method shows the hierarchical model with $\mathrm{R}^{2}$ of $50.87 \%$ and illustrates that the process yield is affected by the concentration of caprylic acid and the amount of time to centrifuge the precipitated plasma but is not by the rotation speed of the centrifugal machine. The secondorder term of caprylic acid is the most significant factor affecting the process yield among all experimental factors.
The predicted process yield by RSM is about $12.97 \%$ when the concentration of caprylic acid and centrifugal time set at $2.82 \%$ and 27.88 (rounded up to 28) minutes, respectively. From this experiment, the rotation speed with a range of 7,500-12,500 $\mathrm{rpm}$ of the centrifugal machine is not significant to the yield. Therefore, the velocity can set at 7,500 rpm for the minimum energy consumption.

\section{Future Work}

Since the process yield in this experiment was calculated from the potency of the centrifugated plasma, not from the concentrated plasma. The additional experiments based on the suggested level should be performed for the scale-up production to confirm if the process yield is improved. Besides the process yield as the interested efficiency in this study, other performances such as the safety of the finished product for humans should investigate before the scale-up production. For example, SDS-PAGE for the purity test to avoid the allergic reaction and the virus inactivation steps to avoid the viral infections from the equine plasma.

\section{Acknowledgement}

This publication has been produced with support from the Queen Saovabha Memorial Institute. This study was also supported by 90th Anniversary of Chulalongkorn University Scholarship.

\section{References}

[1] M. C. D. Santos, M. R. D. Lima, G. C. Furtado, M . D. D. Colletto, T. L. Kipnis, and W. D. D. Silva, "Purification of $F(a b$ ') 2 anti-snake venom by caprylic acid: a fast method for obtaining IgG fragments with high neutralization activity, purity and yield," Toxicon, vol. 27, pp. 297-303, Sep. 1989.

[2] F. Perosa, R. Carbone, S. Ferrone, and F. Dammacco, "Purification of human immunoglobulins by sequential precipitation with caprylic acid and ammonium sulphate," I Immunol Methods, vol. 128, pp. 9-16, Mar. 1990.

[3] G. Rojas, J. M. Jiménez, and J. M. Gutiérrez, "Caprylic acid fractionation of hyperimmune horse plasma: Description of a simple procedure for antivenom production," Toxicon, vol. 32, pp. 351363, Oct. 1994.

[4] S. Eursakun, P. Simsiriwong, and K. Ratanabanangkoon, "Studies on the fractionation of equine antivenom $\operatorname{IgG}$ by combinations of ammonium sulfate and caprylic acid," Toxicon, vol. 60, pp. 1022-1029, Jul. 2012.

[5] P. Simsiriwong, S. Eursakun, and K. Ratanabanangkoon, "A study of the use of caprylic acid and ammonium sulfate in combination for the fractionation of equine antivenom $\mathrm{F}\left(\mathrm{ab}^{\prime}\right)_{2}$," Biologicals, vol. 40, pp. 338-344, May 2012. 
[6] C. Intarapalit and A. Senjuntichai, "Process yields improvement of filter presses in rabies immunoglobulin production," Engineering Journal, vol. 25, pp. 95-103, Mar. 2021.

[7] E. Sloman, "Lubrication," in Plant Engineer's Reference Book, 2nd ed. Oxford, United Kingdom: Butterworth-Heinemann, 2003, pp. 40/1-40/46.

[8] J. Milledge and S. Heaven, "Disc stack centrifugation separation and cell disruption of microalgae. A technical note," Environment and Natural Resources Research, vol. 1, no. 1, pp. 17-24, Nov. 2011.

[9] H. R. Myers, C. D. Montgomery, and C. M. Anderson-Cook, Response Surface Methodology: Process and Product Optimization using Designed Experiments, 4th ed. New Jersey: Wiley, 2016.

[10] T. Sangsawang, N. Rongrat, and A. Yothayuth, "Optimization for biodiesel production by transesterification with electric fields," Engineering Journal, vol. 25, pp. 261-268, Feb. 2021.

[11] M. Santikunaporn, T. Techopittayakul, S. Echaroj, S. Chavadej, Y. H. Chen, M. H. Yuan, and C. Asavatesanupap, "Optimization of biodiesel production from waste cooking oil in a continuous mesoscale oscillatory baffled reactor," Engineering Journal, vol. 24, pp. 19-28, Mar. 2020.

[12] A. Senjuntichai, "Process setting through general linear model and response surface method," IEANG Transactions on Engineering Technologies, vol. 5, pp. $237-$ 248, 2010.

[13] S. Kulpiya and A. Senjuntichai, "Package chip defect reduction on integrated circuit," Applied mechanics and Materials, vols. 462-463, pp. 578-584, 2014.

[14] B. Y. Gajera, D. A. Shah, and H. R. Dave, "Development of an amorphous nanosuspension by sonoprecipitation formulation and process optimization using design of experiment methodology," Int. J Pharmaceutics, vol. 559, pp. 348359, Mar. 2019.

[15] O. Yeniay, "Comparative study of algorithms for response surface optimization," Mathematical and Computational Applications, vol. 19, no. 4, pp. 93-104, 2014.

[16] E. B. M. Barbosa, E. L. F. Senne, and M. B. Silva, "Improving the performance of metaheuristics: An approach combining response surface methodology and racing algorithms," International Journal of Engineering Mathematics, 2019, Article No. 167031.

[17] M. Bashiri and F. Samaei, "Heuristic and metaheuristic structure of response surface methodology in process optimization," 2011 IEEE International Conference on Industrial Engineering and Engineering Management, 6-9 December, 2011, Singapore, pp. 1495-1499.

[18] T. Rakic, I. Kasagic-Vujanovic, M. Jovanovic, B. Jancic-Stojanovic, and D. Ivanovic, "Comparison of full factorial design, central composite design, and Box-Behnken design in chromatographic method development for the determination of Fluconazole and its impurities," Analytical Letters, vol. 47, no. 8, pp. 1334-1347, Feb. 2014.

[19] M. Thianwiboon, "Parameter tuning of autonomous boat in fish farming industry with design of experiment," Engineering Journal, vol. 24, pp. 217-225, Sep. 2020

[20] S. Sitthipong, P. Towatana, S. Lohwirakorn, C. Meengam, and S. Chainarong, "The design of experimental production of briquette solid fuel from plam fiber and kernel meal residual," Engineering Journal, vol. 24, pp. 31-42, Nov. 2020.

[21] S. Khomvilai, S. Daviratanasilpa, D. Pornmuttakun, L. Sakolpap, S. Akesowan, N. Pakmanee, W. Hemmala, K. Limsuwun, T. Tantavichien, and P. Khawplod, "Production of equine rabies immune globulin of high purity, potency, and safety," in Current Laboratory Techniques in Rabies Diagnosis. Research and Prevention: Vol. 2, 1st ed. San Diego: Elsevier, 2015, ch. 24, pp. 293-303.

[22] T. Burnouf, E. Griffiths, A. Padilla, S. Seddik, M. A. Stephano, and J. M. Gutiérrez, "Assessment of the viral safety of antivenoms fractionated from equine plasma," Biologicals, vol. 32, no. 3, pp. 115-128, Sep. 2004.

[23] G. R. Holdich, Fundamental of Particle Technology. Leicestershire, United Kingdom: Midland Information Technology and Publishing, 2002.

[24] F. J. Baker and R. E. Silverton, Introduction to Medical Laboratory Technology, 5th ed. Oxford, United Kingdom: Butterworth-Heinemann, 1976.

[25] D. C. Montgomery, Design and Analysis of Experiments, 10th ed. New Jersey: Wiley, 2019.

[26] G. E. P. Box, J. S Hunter, and W. G. Hunter, Statistics for Experimenters: Design, Innovation and Discovery (Book style), 2nd ed. New Jersey: John Wiley and Sons, 2005.

[27] S. L. C. Ferreira, R. E. Bruns, H. S. Ferreira, G. D. Matos, J. M. David, G. C. Brandao, E. G. P. da Silva, L. A. Portugal, P. S. dos Reis, A. S. Souza, and W. N. I. Dos Santos, "Box-Behnken design: An alternative for the optimization of analytical methods," Analytica Chimica Acta, vol. 597, no. 2, pp. 179-186, Aug. 2007.

[28] M. Dutka, M. Ditaranto, and T. Løvås, "Application of a central composite design for the study of $\mathrm{NOx}$ emission performance of a low NOx burner," Energies, vol. 8, pp. 3606-3627, Aug. 2015.

[29] B. C. Nudel, C. Perdoménico, R. Iácono, and O. Cascone, "Optimization by factorial analysis of caprylic acid precipitation of non-immunoglobulins from hyperimmune equine plasma for antivenom preparation," Toxicon, vol. 59, pp. 68-73, Feb. 2012. 


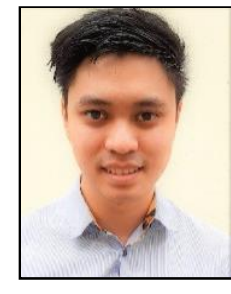

Issada Manohorratad received a bachelor's degree in doctor of pharmacy (Pharm. D.) from faculty of pharmaceutical sciences, Chulalongkorn University, Bangkok, Thailand, in 2018. His major interest is pharmaceutical manufacturing. His aim is to implement the process development in the pharmaceutical manufacturing for the best and ensure the quality of results. In 2019, he has enrolled in master of engineering in industrial engineering (M.Eng.), the department of Industrial Engineering, Chulalongkorn University for acknowledgment of the application of industrial engineering to the pharmaceutical industry.

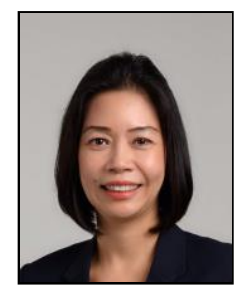

Dr. Angsumalin Senjuntichai obtained her first degree in industrial engineering (B.Eng) from Chulalongkorn University, Bangkok, Thailand in 1994. She received a Master of Science in industrial engineering (M.S.I.E.) from University of Minnesota, Minnesota, USA in 1999, and a Doctoral Degree (D.Eng) in industrial engineering from Asian Institute of Technology, Thailand in 2011.

In 1994, she has joined the department of Industrial Engineering, Chulalongkorn University where she is currently a full-time associate professor.

Dr. Senjuntichai has been constantly involved in professional activities. She has served as a technical consultant to several governments and private sectors for various engineering projects. She has also published papers in international journals and conference proceedings. Her interested fields are applied statistics, financial engineering, and data analytics. 